University of Nebraska - Lincoln

DigitalCommons@University of Nebraska - Lincoln

\title{
On the Validity of the Arrhenius Equation for Electron Attachment Rate Coefficients
}

\author{
Ilya I. Fabrikant \\ University of Nebraska-Lincoln, ifabrikant@unl.edu \\ Harmut Hotop \\ Universität Kaiserslautern, hotop@physik.uni-kl.de
}

Follow this and additional works at: https://digitalcommons.unl.edu/physicsfabrikant

Part of the Physics Commons

Fabrikant, llya I. and Hotop, Harmut, "On the Validity of the Arrhenius Equation for Electron Attachment Rate Coefficients" (2008). Ilya Fabrikant Publications. 4.

https://digitalcommons.unl.edu/physicsfabrikant/4

This Article is brought to you for free and open access by the Research Papers in Physics and Astronomy at DigitalCommons@University of Nebraska - Lincoln. It has been accepted for inclusion in llya Fabrikant Publications by an authorized administrator of DigitalCommons@University of Nebraska - Lincoln. 


\title{
On the validity of the Arrhenius equation for electron attachment rate coefficients
}

\author{
Ilya I. Fabrikant ${ }^{1, a)}$ and Hartmut Hotop ${ }^{2}$ \\ ${ }^{1}$ Department of Physics and Astronomy, University of Nebraska, Lincoln, Nebraska 68588-0111, USA \\ ${ }^{2}$ Fachbereich Physik, Universität Kaiserslautern, D-67653 Kaiserslautern, Germany
}

(Received 11 December 2007; accepted 15 January 2008; published online 27 March 2008)

\begin{abstract}
The validity of the Arrhenius equation for dissociative electron attachment rate coefficients is investigated. A general analysis allows us to obtain estimates of the upper temperature bound for the range of validity of the Arrhenius equation in the endothermic case and both lower and upper bounds in the exothermic case with a reaction barrier. The results of the general discussion are illustrated by numerical examples whereby the rate coefficient, as a function of temperature for dissociative electron attachment, is calculated using the resonance $R$-matrix theory. In the endothermic case, the activation energy in the Arrhenius equation is close to the threshold energy, whereas in the case of exothermic reactions with an intermediate barrier, the activation energy is found to be substantially lower than the barrier height. (c) 2008 American Institute of Physics.
\end{abstract}

[DOI: $10.1063 / 1.2841079]$

\section{INTRODUCTION}

The rate coefficient for reactive processes between two colliding species is defined by $k=\left\langle\sigma_{n}(v) v\right\rangle$, i.e., by the (thermal) average of the product of the state-dependent reaction cross section $\sigma_{n}(v)$ (n denoting the internal states of the species) times the relative collision velocity $v$. It is well known that the temperature dependence of the rate coefficient for many chemical reactions is described by the Arrhenius equation ${ }^{1,2}$

$$
k(T)=A e^{-E_{a} / T},
$$

where $T$ is the temperature in energy units, $A$ is a constant, and $E_{a}$ is the so-called activation energy. In numerous cases, activation energies have thus been determined from plots of $\ln k$ versus $1 / T$. In general, the prefactor $A$ might be temperature dependent too but this dependence is normally much weaker than exponential.

In the present paper, we investigate the temperature dependence of a special class of reactive collisions, namely, of the dissociative electron attachment (DEA) reaction (for a recent review see Ref. 3)

$$
\mathrm{XY}+e \rightarrow \mathrm{X}+\mathrm{Y}^{-},
$$

where $\mathrm{X}$ and $\mathrm{Y}^{-}$are neutral and ionic fragments, not necessarily monoatomic.

Many of these reactions studied experimentally were found to obey the Arrhenius equation (see, e.g., Refs. 4-20), albeit over rather limited temperature ranges. If we assume that the electrons (kinetic temperature $T_{e}$ ) and the target molecules (internal temperature $T_{G}$ ) are in thermal equilibrium corresponding to the temperature $T=T_{e}=T_{G}$, then the general expression for the rate coefficient is given by (in the following, we ignore the rotational degrees of freedom)

\footnotetext{
${ }^{a)}$ Electronic mail: ifabrikant1@unl.edu.
}

$$
k(T)=\frac{1}{S} \sum_{n} e^{-\epsilon_{n} / T} \int v \sigma_{n}(v) f(v, T) d v,
$$

where $\sigma_{n}(v)$ is the DEA cross section for a given initial vibrational state $n$ as a function of the electron velocity $v, \epsilon_{n}$ is the vibrational energy of the target molecule (measured from the bottom of the potential), $S$ is the partition function

$$
S=\sum_{n} e^{-\epsilon_{n} / T},
$$

and $f$ is the Maxwellian distribution function

$$
f(v, T)=\left(\frac{2}{\pi}\right)^{1 / 2}\left(\frac{m}{T}\right)^{3 / 2} v^{2} \exp \left(-\frac{m v^{2}}{2 T}\right) .
$$

In the present work, we treat direct DEA processes, i.e., we do not include molecules such as $\mathrm{SF}_{6}$ for which the primary electron capture process is followed by the formation of a long-lived anion via intramolecular redistribution; ${ }^{3,10,13,17,20,21}$ such systems need a detailed treatment of the postattachment evolution of the anion by kinetic modeling (see, e.g., Ref. 20). In general, $n$ in Eq. (3) stands for all quantum numbers representing vibrations of the molecule. However, we will assume that the cross section $\sigma$ depends essentially only on one vibrational quantum number, corresponding to vibrational motion along the reaction coordinate, that is, $\mathrm{X}-\mathrm{Y}$ stretch. Note that the electron velocity is much higher than the molecular velocity and, thus, the relative collision velocity $v$ is simply given by the electron velocity.

The variation of $k(T)$ with temperature is determined by the dependence of the cross section on $n$ and $v$. The Arrhenius equation is usually associated with a reaction barrier which might be due to either the endothermicity of the DEA process or due to a barrier separating the initial $X Y$ state from the exothermic final $\mathrm{X}+\mathrm{Y}^{-}$state. In the endothermic case, we assume that the activation energy is identical with the reaction threshold. In the exothermic case, the reaction 


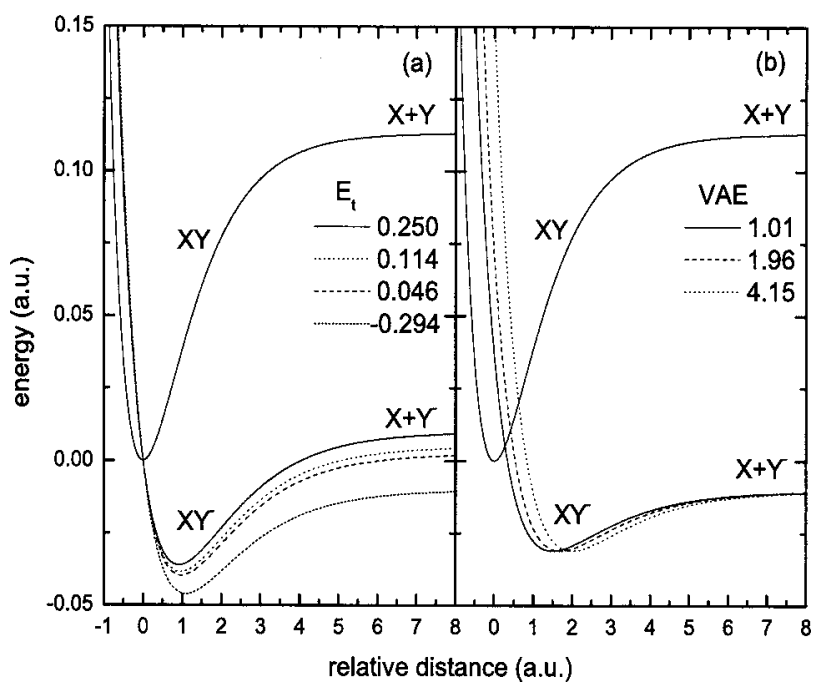

FIG. 1. Potential energy curves for the neutral and anion systems relevant for the considered dissociative electron attachment processes. Panel (a): cases with no intermediate barrier (see Table I). The corresponding reaction thresholds $E_{t}$ are given in $\mathrm{eV}$. Panel (b): cases with intermediate barrier (see Table III); the corresponding vertical attachment energies are listed in $\mathrm{eV}$.

barrier is usually associated with the energy of the crossing point between the neutral and anion potential curves. The two situations are illustrated in Fig. 1.

Panel (a) represents three endothermic cases with no intermediate barrier and one exothermic case with a negative reaction threshold $E_{t}$. All cases are characterized by a favorable Franck-Condon factor for the transition from the vibrational ground state of the neutral molecule to the anion state at low electron energies. Practical examples for case (a) include $\mathrm{XY}=\mathrm{HI}$ (slightly exothermic), ${ }^{22-24}$ and DI (slightly endothermic), ${ }^{22,24}$ and the exothermic cases $\mathrm{CH}_{3} \mathrm{I}$ (Refs. 16 and 25) and $\mathrm{CF}_{3} \mathrm{I}^{26,27}$ In the exothermic case with an intermediate barrier [panel (b)], the vibrational states of the neutral molecule lying below the energy of the crossing point are expected to have small DEA cross sections. This happens because for these vibrational states at low electron energies the Franck-Condon overlap between the neutral and anion states is small, and for higher energies, the electron capture occurs far from the crossing point, and the intermediate anion state is likely to decay before reaching the crossing (stabilization) point. Therefore, it is usually assumed that the activation energy is identical with or close to the reaction barrier, i.e., the difference $E_{B}$ between the energy of the crossing point and the energy of the neutral ground vibrational state. Examples for case (b) include the molecules $\mathrm{CH}_{3} \mathrm{Br},{ }^{16,19} \mathrm{CH}_{3} \mathrm{Cl},{ }^{16,28}$ and $\mathrm{CF}_{3} \mathrm{Y}(\mathrm{Y}=\mathrm{Cl}, \mathrm{Br}){ }^{18,29,30}$ As a special example, we present in Fig. 2 an Arrhenius plot of the DEA rate coefficient for the $\mathrm{CF}_{3} \mathrm{Br}$ molecule. ${ }^{18}$ Ignoring details of the experimental observations (symbols) and of the theoretical results (full curve), one observes that the validity of the Arrhenius equation is certainly limited toward low temperatures and probably toward high temperatures as well. Because of these limitations, the question arises as to what is the relation between the activation energy and the reaction barrier. Indeed, the slope calculated from the exponential part of the theoretical curve in Fig. 2 yields an activation energy of $E_{a}=52 \mathrm{meV}$, which is substantially lower than the

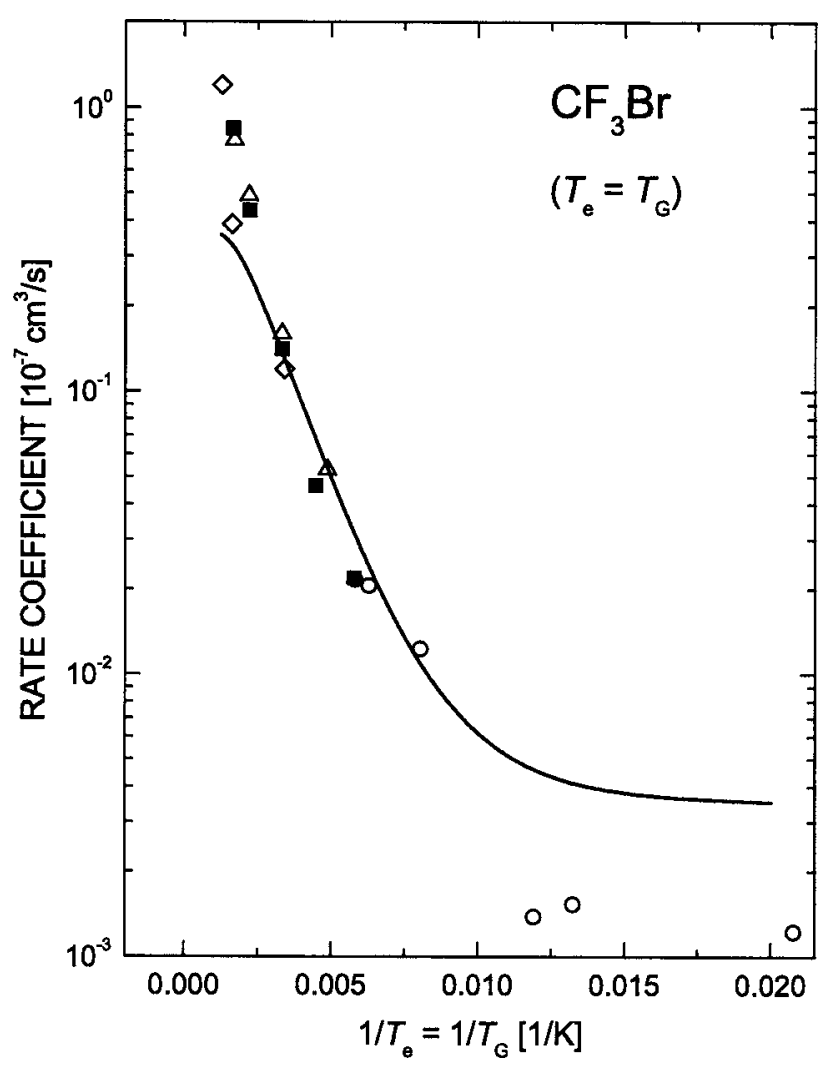

FIG. 2. Dependence of the thermal rate coefficient on temperature for DEA to $\mathrm{CF}_{3} \mathrm{Br}$. Full curve: $R$-matrix theory (Ref. 18). Symbols denote different swarm experiments: open triangles (Ref. 7), open diamonds (Ref. 11), open circles (Ref. 15), and full squares (Ref. 18) (the data point at the lowest temperature, $T=173 \mathrm{~K}$, was given incorrectly in Fig. 15 of Ref. 18).

reaction barrier $E_{B}=120 \mathrm{meV}$. A similar situation occurs for DEA to $\mathrm{CH}_{3} \mathrm{Br}^{19}$ the theoretical value of $E_{a}=249 \mathrm{meV}$, which agrees with several measurements, is substantially lower than the reaction barrier $E_{B}=372 \mathrm{meV}$.

In what follows, we will analyze endothermic and exothermic cases in more detail and discuss specific examples. We will be particularly interested in the relation between the activation energy and the reaction threshold (in the former case) or the reaction barrier (in the latter case).

\section{GENERAL CONSIDERATIONS}

\section{A. Endothermic case}

Consider first the endothermic case with no intermediate barrier [three upper anion curves in Fig. 1(a)]. The cross section as a function of $n$ and $v$ has the form

$$
\sigma_{n}(v)=s_{n} \theta\left(v-v_{n}\right),
$$

where $\theta(x)$ is the step function, $v_{n}$ is the threshold velocity determined from the conservation of energy

$$
\frac{m v_{n}^{2}}{2}=E_{t}-\epsilon_{n}+\epsilon_{0},
$$

where $E_{t}$ is the threshold energy for attachment to the ground vibrational state, and $s_{n}$ denotes a cross section which depends weakly, that is, nonexponentially, on $v$. If the anion curve crosses the neutral curve in the region close to the equilibrium internuclear separation for the neutral, as pre- 
sented in Fig. 1(a), the Franck-Condon overlap is favorable for low $n$ and depends weakly on $n$ and on the velocity $v$. Thus, the dependence of $s_{n}$ on $n$ is weak as well. However, for unfavorable curve crossing, the $n$ dependence of $s_{n}$ is strong $^{31,32}$ (see also the more recent Ref. 24). Also, at much higher energies the energy dependencies of the FranckCondon and survival factors become strong, and $\sigma$ drops with $v$ at least exponentially. This behavior affects the hightemperature region, as will be illustrated below.

Using Eq. (6), we can estimate the partial attachment rate coefficient for a given electron temperature $T_{e}$ as a function of $n$ for $v_{n}^{2}>0$,

$$
k\left(n, T_{e}\right)=\int v \sigma_{n}(v) f\left(v, T_{e}\right) d v=k_{n} \exp \left(-m v_{n}^{2} / 2 T_{e}\right),
$$

where $k_{n}$ is a rate coefficient which weakly (nonexponentially) depends on $T_{e}$. Using the harmonic approximation for $\epsilon_{n}, \epsilon_{n}-\epsilon_{0}=n \omega$, where $\omega$ is the vibrational frequency (in energy units), we obtain

$$
k\left(n, T_{e}\right)=k_{n} \exp \left(-\frac{E_{t}-n \omega}{T_{e}}\right), \quad E_{t}>n \omega .
$$

For $n \omega>E_{t}$ (exothermic case), we assume that the rate coefficient is given by a rate coefficient $k_{c}$ weakly dependent on $n$ and $T_{e}$.

We define now $n_{0}$ such that $n_{0} \omega<E_{t}<\left(n_{0}+1\right) \omega$. Then, $E_{t}=\left(n_{0}+p\right) \omega$, where $0<p<1$. For the total attachment rate coefficient, Eq. (3) $\left(T_{e}=T_{G} \equiv T\right)$,

$$
k(T)=\frac{1}{S} \sum_{n} k(n, T) \exp (-n \omega / T),
$$

we obtain

$$
k(T)=\frac{1}{S}\left[\sum_{n=0}^{n_{0}} k_{n} e^{-E_{t} / T}+k_{c} \sum_{n=n_{0}+1}^{\infty} e^{-n \omega / T}\right]
$$

or

$$
k(T)=e^{-E_{t} / T}\left[p\left(n_{0}\right)\left(1-e^{-\omega / T}\right)+k_{c} e^{-(1-p) \omega / T}\right],
$$

where

$$
p\left(n_{0}\right)=\sum_{n=0}^{n_{0}} k_{n}
$$

If $T \ll \omega$, we recover the Arrhenius equation with the activation energy $E_{a}=E_{t}$. For higher temperatures, we should expect certain deviations due to the second and third exponentials in Eq. (12). Also, at high temperatures our assumption about the weak dependence of $s_{n}$ on velocity $v$ is not valid any longer.

As a sideline we mention that Arrhenius-type behavior with $E_{t} \approx E_{a}$ also holds for a nonequilibrium situation when the electron temperature $T_{e}$ is much smaller than the gas temperature $T_{G}$. Such a situation is met, for example, for Rydberg electron transfer (RET) collisions involving atoms with high principal quantum numbers. In this case, the partial rate coefficient $k(n ; \mathrm{RET})$ is zero for $n \leqslant n_{0}$ and has an essentially constant value $k_{0}$ for $n>n_{0}$. Thus,

$$
\begin{aligned}
k\left(T=T_{G} ; \mathrm{RET}\right) & =\left(k_{0} / S\right) \sum_{n=n_{0}+1}^{\infty} \exp (-\omega n / T) \\
& =k_{0} \exp \left[-\left(n_{0}+1\right) \omega / T\right] \\
& \approx k_{0} \exp \left(-E_{t} / T\right)
\end{aligned}
$$

Deviations from the Arrhenius equation with a constant activation energy may also arise from rotational effects. To demonstrate this, we assume, for the sake of simplicity, that only the ground vibrational state is populated, although a more general treatment which includes both rotational and vibrational motion is possible. With inclusion of rotational motion only and assuming again the pseudodiatomic case, we have

$$
k(T)=\frac{1}{S_{\text {rot }}} \sum_{J}(2 J+1) e^{-\mathcal{E}_{J} / T} k(J, T),
$$

where $S_{\text {rot }}$ is the partition function for rotational motion, $\mathcal{E}_{J}$ is the rotational energy, and $k(J, T)$ is the partial rate coefficient for a given $J$. With the same assumption as we have made for $k(n, T)$

$$
k(J, T)=k_{J} \exp \left(-\frac{E_{t}-\mathcal{E}_{J}}{T}\right), \quad E_{t}>\mathcal{E}_{J},
$$

and for $\mathcal{E}_{J}>E_{t}$, the rate coefficient has a constant value $k_{c}$.

For further estimates, we will use the classical approximation for rotational motion, that is, we will treat $J$ as a continuous variable and use $\mathcal{E}_{J}=B_{\text {rot }} J^{2}$, where $B_{\text {rot }}$ is the rotational constant. Computations show that this approximation works quite well even at temperatures as low as $200 \mathrm{~K}$ for such a light molecule as $\mathrm{HCl}$. Then, for the partition function, we obtain $S_{\text {rot }}=T / B_{\text {rot }}$, and for the rate coefficient

$$
k=\left(t\left(J_{0}\right) \frac{B}{T}+k_{2}\right) e^{-E_{t} / T}, \quad t\left(J_{0}\right)=\sum_{J=0}^{J_{0}} k_{J}(2 J+1) .
$$

The temperature dependence of the preexponential factor indicates that the effective activation energy varies with temperature because of the rotational motion. A rigorous study of this effect requires one to account for the energy dependence of the DEA cross section above the threshold because this dependence affects the preexponential factor as well, but qualitatively, it is clear that rotational motion lowers the effective activation energy at higher temperatures. This is quantitatively confirmed by theoretical results for the temperature-dependent rate coefficients for endothermic DEA to hydrogen halides using the nonlocal resonance model $^{24}$ (see Sec. IV A).

\section{B. Exothermic case with intermediate barrier}

In contrast to the endothermic case, the cross section is nonzero for any $n$ and $v$. However, it is substantially suppressed at energies below the reaction barrier. The associated partial rate coefficient $k\left(n ; T_{e}\right)$ grows strongly with $n$ below the barrier but is rather weakly dependent on electron temperature $T_{e}$. It is quite realistic-as supported by test calculations - to use the approximate relation, especially at rather low $T_{e}$ or for RET processes 


$$
k\left(n, T_{e}\right)=k_{1} e^{\alpha n},
$$

with $k_{1}, \alpha$ constants, at $n \omega<E_{B}$. At $n \omega>E_{B}$, we assume that $k\left(n, T_{e}\right)$ equals a constant value $k_{2}$.

For the total attachment rate coefficient, Eq. (3), we obtain

$$
k(T)=\frac{1}{S}\left[k_{1} \sum_{n=0}^{n_{0}} e^{\alpha n-n \omega / T}+k_{2} \sum_{n=n_{0}+1}^{\infty} e^{-n \omega / T}\right],
$$

where $n_{0}$ is the integral part of $E_{B} / \omega$. Finally,

$$
k(T)=k_{1} \frac{\left[1-e^{(\alpha-\omega / T) n_{0}}\right]\left[1-e^{-\omega / T}\right]}{1-e^{\alpha-\omega / T}}+k_{2} e^{-\left(n_{0}+1\right) \omega / T} .
$$

The Arrhenius-type dependence is obtained by making the following approximate assumptions:

$$
\frac{\omega}{T}>1, \quad \alpha-\frac{\omega}{T}>2,
$$

or

$$
1<\frac{\omega}{T}<\alpha-2
$$

Then, $k(T)=k_{1}^{\prime} e^{-A / T}$, where $k_{1}^{\prime}=k_{1} \exp \left[\left(n_{0}-1\right) \alpha\right], \quad E_{a}=\left(n_{0}\right.$ $-1) \omega$.

This estimate is rather conservative, and some practical cases discussed in Sec. IV B have values of $\alpha$ close to 3, while the Arrhenius equation is still valid, albeit in a rather limited range. Apparently, the range of the validity of the Arrhenius equation in the exothermic case with a barrier is much narrower than in the endothermic case. Moreover, the activation energy is somewhat lower than the reaction barrier.

\section{R-MATRIX THEORY AND CALCULATIONAL PROCEDURE}

Dissociative electron attachment to many molecules, particularly of the type $\mathrm{CX}_{3} \mathrm{Y}$, where $\mathrm{X}$ stands for the $\mathrm{H}$ or $\mathrm{F}$ atom and $\mathrm{Y}$ for a halogen atom, in the low-energy region occurs through electron capture into the lowest unoccupied molecular orbital of $a_{1}$ symmetry yielding the products $\mathrm{CX}_{3}$ and $\mathrm{Y}^{-}$.

To calculate dissociative attachment cross sections, we employ the resonance $R$-matrix theory. ${ }^{33}$ The $R$-matrix, or the reciprocal logarithmic derivative of the electron wave function on the surface of the $R$-matrix sphere, in the fixednuclei approximation has the form

$$
R(\rho)=\frac{\gamma^{2}(\rho)}{W(\rho)-E}+R_{b},
$$

where $\rho$ is the reaction coordinate relative to the equilibrium separation, $W(\rho)$ is the lowest $R$-matrix pole, $\gamma(\rho)$ is the surface amplitude, and $R_{b}$ is a background term independent of $\rho$ and electron energy $E$. The physical significance of $W(\rho)$ is that it represents the energy of the resonance state. The diabatic anion potential $U(\rho)$ can be written as $[V(\rho)$ denotes the potential energy of the neutral molecule]

$$
U(\rho)=W(\rho)+V(\rho) .
$$

The surface amplitude $\gamma$ is typically a slowly varying function of $\rho$ and can be considered as a constant. However, to introduce more flexibility in the theory, we parametrize it in the following form:

$$
\gamma(\rho)=\frac{\gamma_{0}}{e^{\zeta \rho}+a} .
$$

Parameters $\gamma_{0}, \zeta$, and $a$ are usually adjusted empirically to reproduce the absolute values of attachment rate coefficients from swarm measurements. In the present model calculations, all parameters determining the surface amplitude $\gamma(\rho)$ were chosen the same as in the $\mathrm{CF}_{3} \mathrm{Br}$ calculations. ${ }^{18}$

To calculate DEA cross sections, we incorporate nuclear dynamics and solve basic equations of the resonance $R$-matrix theory in the quasiclassical approximation. ${ }^{33}$ As part of this procedure, we calculate electron scattering wave functions outside the $R$-matrix sphere that include dipolar and polarization interactions. We have chosen $\mathrm{CF}_{3} \mathrm{Br}$ as a typical case and used the same dipole moment and polarizability of the target as in Ref. 18, that is, for the dipole moment $0.65 \mathrm{D}=0.256$ a.u. and for the polarizability 45 a.u.

\section{MODEL CALCULATIONS OF DEA CROSS SECTIONS}

\section{A. Cases with no intermediate barrier}

To illustrate the case with no intermediate barrier, we have chosen several anion curves of the type presented in Fig. 1(a). The neutral potential curve describing the stretching motion along the reaction coordinate is parametrized in the Morse form

$$
V(\rho)=D_{0}\left(e^{-\alpha \rho}-1\right)^{2},
$$

where $\rho$ is the reaction coordinate relative to the equilibrium distance, $D_{0}=3.08 \mathrm{eV}$, and $\alpha=0.8728$ a.u.

For the anion curve, we use the parametrization

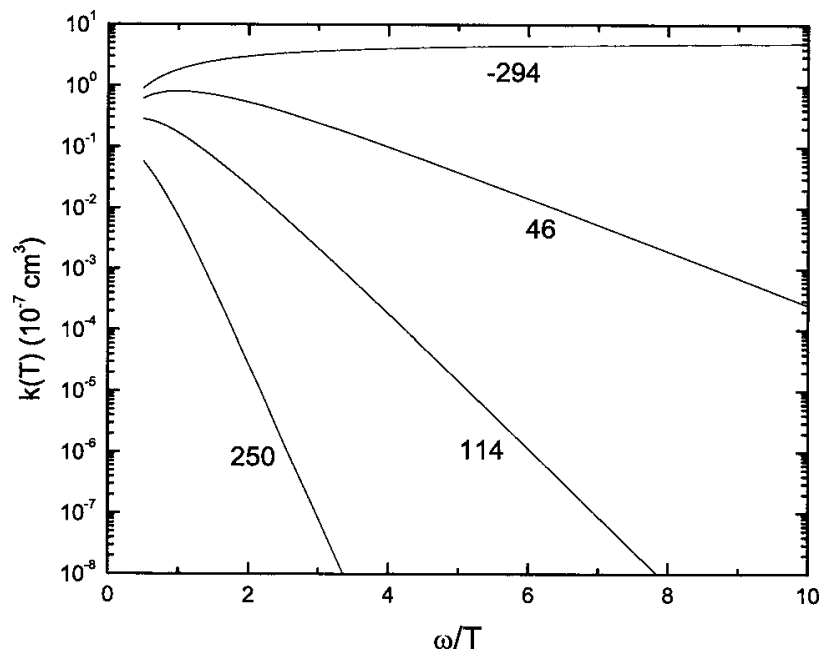

FIG. 3. DEA rate coefficients as functions of $1 / T$ in units of vibrational quantum $(\omega=43.4 \mathrm{meV})$ for cases represented by the potential curves of Fig. 1. The corresponding reaction thresholds in $\mathrm{meV}$ are indicated for each curve. 
TABLE I. Energetic parameters for cases with no intermediate barrier [see Fig. 1(a)]; the vibrational frequency is fixed at the value $\omega=43.3 \mathrm{meV}$

\begin{tabular}{ccccc}
\hline \hline$C(\mathrm{eV})$ & $D(\mathrm{meV})$ & $E_{t}(\mathrm{meV})$ & $T_{u}(\mathrm{~K})$ & $E_{a}(\mathrm{meV})$ \\
\hline 4.732 & 272 & 250 & 350 & 252 \\
4.596 & 136 & 114 & 140 & 110 \\
4.528 & 68 & 46 & 120 & 45 \\
4.188 & -272 & -294 & & \\
\hline \hline
\end{tabular}

$$
U(\rho)=B e^{-2 \beta \rho}-C e^{-\beta \rho}+D,
$$

with $B=4.61 \mathrm{eV}$ and $\beta=0.6918$ a.u. These values were used in Ref. 18 to describe DEA to the $\mathrm{CF}_{3} \mathrm{Br}$ molecule. The actual values of $C$ and $D$ for $\mathrm{CF}_{3} \mathrm{Br}$ used in Ref. 18 are $C$ $=3.167 \mathrm{eV}$ and $D=-0.283 \mathrm{eV}$. In the present work, we vary them to see the effect of endothermicity on the $k(T)$ dependence, but we keep the crossing point between the neutral and anion curve fixed at the equilibrium internuclear separation for the neutral, as shown in Fig. 1(a). This makes the Franck-Condon overlap favorable for the electron capture into the anion state, and makes the DEA cross section large and relatively weakly (nonexponentially) dependent on energy just above the threshold.

In order to investigate how the attachment rate coefficients depend on the threshold energy $E_{t}$, we chose several asymptotic values of the anion curves. To contrast the rate coefficient behavior for endothermic reactions with the exothermic case, one curve corresponds to a negative threshold. In the latter case, $k(n, T)$ depends weakly on $n$, which results in a simple $T^{-1 / 2}$ dependence of the total rate coefficient. In Fig. 3, we present the attachment rate coefficients $k(T)$ on a semilog plot. As expected, the Arrhenius equation holds for $\omega / T \gg 1$, but the range of validity grows with the growth of the threshold energy $E_{t}$, down to $\omega / T \approx 1$ for $E_{t} / \omega \approx 6(\omega$ $=43.3 \mathrm{meV}$, as for the $\mathrm{CF}_{3} \mathrm{Br}$ case $\left.{ }^{18}\right)$. To make a rough estimate for the upper temperature bound $T_{u}$ for the validity of the Arrhenius equation, we determined from Fig. 3 the parameter $\omega / T_{u}$ such that for $\omega / T<\omega / T_{u}$ the deviation of the actual curve from the straight-line behavior becomes substantial.

In Table I, we present the anion curve parameters, threshold energies, the upper temperature bound $T_{u}$, and the activation energies. The activation energies, taken from the slopes of the curves in Fig. 3 within the "Arrhenius range," are very close to the reaction thresholds.

The behavior of the total rate coefficient $k(T)$ is consistent with the behavior of the partial rate coefficient $k\left(n, T_{e}\right)$ given by Eq. (9). In Fig. 4, we present $k\left(n, T_{e}\right)$ as a function of $n$ for several electron temperatures, as calculated for the case $E_{t}=114 \mathrm{meV}$. Although this dependence is not exactly linear on the semilog scale, the average slope varies inversely proportional to $T_{e}$, as predicted by Eq. (9).

The influence of the rotational motion is demonstrated by the results of Houfek et al. ${ }^{24}$ who calculated temperaturedependent rate coefficients for DEA to hydrogen halides (Table II in Ref. 24). As an example, we discuss $\mathrm{HCl}$ here. The threshold energy in this case is $0.821 \mathrm{eV}$. Calculation of the activation energy from the rate coefficients gives $E_{a}$ $=0.797 \mathrm{eV}$ in the temperature range of $100-200 \mathrm{~K}$ and $E_{a}$

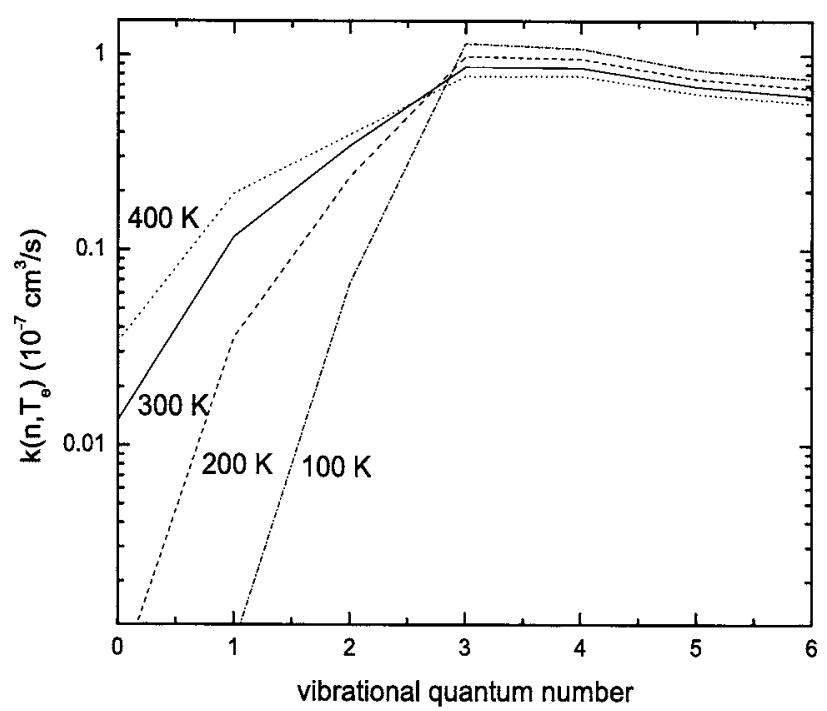

FIG. 4. Partial rate coefficients as functions of vibrational quantum number $n$ for endothermic DEA with a reaction threshold $114 \mathrm{meV}$ and the vibrational quantum number $\omega=43.3 \mathrm{meV}$, calculated for four electron temperatures $T_{e}$.

$=0.711 \mathrm{eV}$ in the temperature range of $900-1000 \mathrm{~K}$. This confirms our discussion in Sec. II A, showing that rotational effects lead to a decrease of the effective activation energy at higher temperatures. Activation energies derived from the rate coefficients for other hydrogen halides presented by Houfek et al. ${ }^{24}$ show a similar behavior.

\section{B. Exothermic case with reaction barrier}

To study the exothermic reaction case including an intermediate barrier, we first choose the potential curve parameters describing DEA to $\mathrm{CF}_{3} \mathrm{Br}$; ${ }^{18}$ in this case the "classical barrier height" $E_{C}$, i.e., the energy of the crossing point above the minimum of the neutral potential curve amounts to $E_{C}=142 \mathrm{meV}$ and the barrier energy $E_{B}=E_{C}-\omega / 2$ to $120.4 \mathrm{meV}$. We vary the vibrational quantum $\omega$ by changing

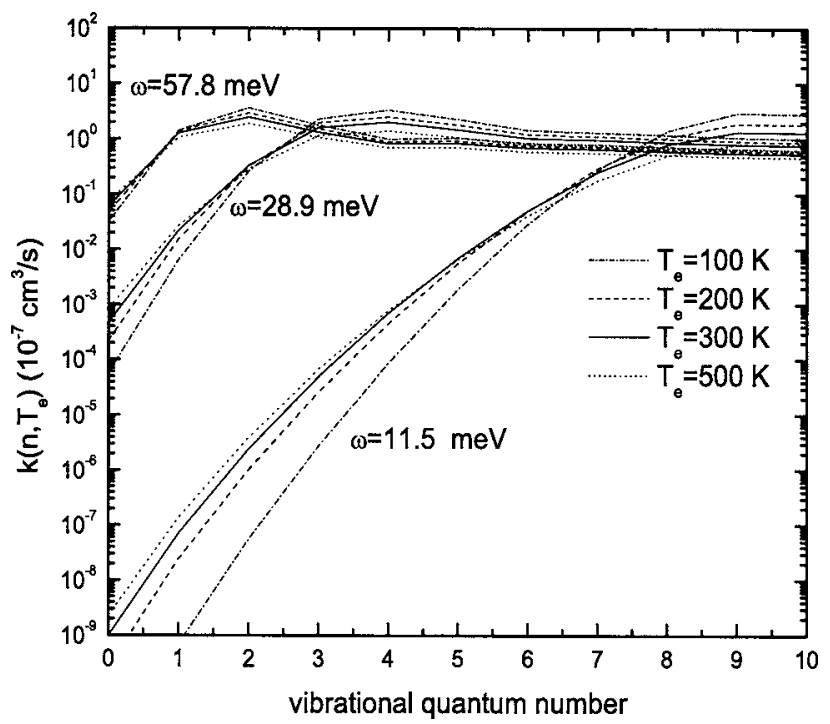

FIG. 5. Partial rate coefficients $k\left(n, T_{e}\right)$ for exothermic DEA with a classical intermediate barrier $E_{C}=142 \mathrm{meV}$, calculated for four electron temperatures and three vibrational frequencies. 


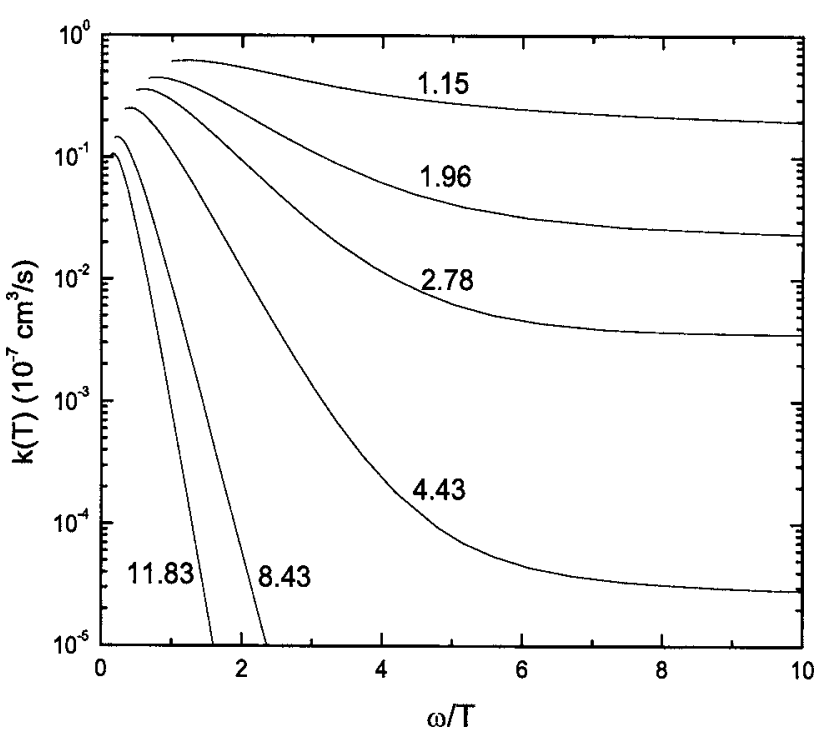

FIG. 6. Dependence of DEA rate coefficients on $\omega / T$ for potential energy curves involving a classical barrier height $E_{C}=142 \mathrm{meV}$, as calculated for six vibrational frequencies between 11.5 and $86.0 \mathrm{meV}$ (see Table II). The associated graphs are labeled by the ratios $E_{B} / \omega\left(E_{B}=E_{C}-\omega / 2\right)$.

the reduced mass of the molecule. The case $\omega=43.3 \mathrm{meV}$ $\left(E_{B} / \omega=2.78\right)$ corresponds to the frequency of the symmetric stretch vibration $\nu_{3}$ of $\mathrm{CF}_{3} \mathrm{Br}$.

In Fig. 5, we present the partial rate coefficients $k\left(n, T_{e}\right)$ as a function of $n$ for three frequencies $\omega(11.5,28.9$, and $57.8 \mathrm{meV})$ and for four electron temperatures $T_{e}(100,200$, 300 , and $500 \mathrm{~K}$ ). In accord with our assumption, Eq. (18), an almost exponential rise with increasing $n$ is observed for vibrational levels $n$ with energies not too close to the classical barrier. The slope $\alpha$ has values between 2.5 and 3.5 at these low $n$ and decreases rather weakly with rising electron temperature. For levels $n$ above a certain value (starting at energies significantly below the classical barrier), the rate coefficients are almost independent of $n$. This observation is associated with the quantum effects near the top of the barrier: the vibrational wave function of the initial state is nonzero in the classically forbidden region, and this makes the vertical Franck-Condon transition possible even at energies below the reaction barrier.

In the thermally averaged rate coefficient $k(T)$, the contribution from each level $n$ is obtained by weighing the partial rate coefficients in Fig. 5 with the factor $\exp (-n \omega / T)$. In Fig. 6, we present the attachment rate coefficients as a function of $\omega / T$ for six values of $\omega$; the curves are labeled by the ratio $E_{B} / \omega$. In all cases, deviations from Arrhenius-type exponential behavior are observed at both low and high $\omega / T$. At high $\omega / T$ (low-temperature limit), the rate coefficients turn to nearly constant values which basically reflect the rate coefficients for the $n=0$ vibrational level at low electron temperature (or for RET processes). At low $\omega / T$ (hightemperature limit), a substantial fraction of the electron distribution function possesses velocities above the threshold velocity for levels $n$ below the barrier, and this leads to a levelling off of the exponential rise toward higher temperatures. At even higher temperatures (not included in Fig. 6), the electron distribution function progressively reaches into the range where the cross sections rapidly decrease with rising energies (as $1 / E$ or even faster); correspondingly, the rate coefficients turn over and decrease toward very low $\omega / T$.

The activation energies, estimated from the curves in Fig. 6 in the intermediate $\omega / T$ range, are always substantially smaller than the barrier energy $E_{B}$. This can be seen in Table II where we present lower and upper temperature bounds, $T_{l}$ and $T_{u}$, for the validity of the Arrhenius equation, and activation energies derived from the semilog plots of Fig. 6. Estimates for $T_{l}$ and $T_{u}$ were obtained in the same way as for the endothermic case (Sec. IV A). The ratios $E_{a} / E_{B}$ decrease monotonically from $0.61 \quad(\omega=11.5 \mathrm{meV})$ to $0.24 \quad(\omega$ $=86.0 \mathrm{meV}$ ). For high $\omega$ (low reduced mass), the system differs most strongly from the classical expectation. The amplitude of the vibrational wave function is extended more (larger de Broglie wavelength) and spreads further into the classically forbidden region. Thus, the Franck-Condon factor relevant for, e.g., DEA to $n=0$ is largest for high $\omega$ and leads to rather high values of the rate coefficients even at large $\omega / T$ values. Correspondingly, the slope of the interconnecting exponential section (which is proportional to the activation energy) is reduced for higher $\omega$.

The case with $\omega=43.3 \mathrm{meV}, E_{C}=142 \mathrm{meV}$ corresponds to that of DEA to $\mathrm{CF}_{3} \mathrm{Br}$, as discussed in detail in Ref. 18 . Experimental studies on the temperature dependence of the DEA rate coefficient for $\mathrm{CF}_{3} \mathrm{Br}$ in Refs. 7, 11, 15, and 18 (see Fig. 2) yielded activation energies of $75-80 \mathrm{meV}$, about $50 \%$ higher than the calculated value. This difference between the calculated value of $52 \mathrm{meV}$ and the experimental values was tentatively attributed to the possibility that vibrational modes other than the $\mathrm{C}-\mathrm{Br}$ stretching mode $\nu_{3}$ may play an active role in the DEA process. We note that the one-dimensional model was found to describe DEA to, e.g., the methyl halide $\mathrm{CH}_{3} \mathrm{Br}$ (exothermic with a barrier of about

TABLE II. Energetic parameters for the exothermic case with a fixed intermediate barrier $\left(E_{C}=142 \mathrm{meV}, E_{B}\right.$ $\left.=E_{C}-\omega / 2\right) . E_{a}$ denotes the activation energy deduced from the exponential slope of the calculated rate coefficient (see Fig. 6). $T_{l}$ and $T_{u}$ represent the lower and upper temperature limits of Arrhenius behavior.

\begin{tabular}{cccccc}
\hline \hline$\omega(\mathrm{meV})$ & $T_{l}(\mathrm{~K})$ & $T_{u}(\mathrm{~K})$ & $E_{B} / \omega$ & $E_{B}(\mathrm{meV})$ & $E_{a}(\mathrm{meV})$ \\
\hline 11.5 & 50 & 170 & 11.83 & 136 & 83.1 \\
15.9 & 60 & 207 & 8.43 & 134 & 78.1 \\
28.9 & 110 & 380 & 4.43 & 128 & 65.3 \\
43.3 & 170 & 410 & 2.78 & 120 & 52.2 \\
57.8 & 220 & 460 & 1.96 & 113 & 42.2 \\
86.0 & 330 & 540 & 1.15 & 99 & 23.7 \\
\hline \hline
\end{tabular}


TABLE III. Parameters for exothermic case involving intermediate barriers with different classical barrier heights $\left(E_{C}=72-550 \mathrm{meV}\right)$ and vertical attachment energies (VAE), but fixed vibrational frequency $(\omega=43.3 \mathrm{meV}$; $\left.E_{B}=E_{C}-\omega / 2\right) . E_{a}$ denotes the activation energy deduced from the exponential slope of the calculated rate coefficient (see Fig. 7).

\begin{tabular}{ccccc}
\hline \hline$\rho_{s}$ (a.u.) & VAE $(\mathrm{eV})$ & $E_{B} / \omega$ & $E_{B}(\mathrm{meV})$ & $E_{a}(\mathrm{meV})$ \\
\hline-0.12 & 0.59 & 1.15 & 50 & $\approx 3$ \\
0.0 & 1.01 & 2.78 & 120 & 52 \\
0.1 & 1.45 & 4.39 & 190 & 109 \\
0.2 & 1.96 & 6.17 & 267 & 177 \\
0.3 & 2.58 & 8.08 & 350 & 252 \\
0.5 & 4.15 & 12.19 & 528 & 419 \\
\hline \hline
\end{tabular}

$350 \mathrm{meV}$ ) well, ${ }^{16,19}$ while deficiencies were observed in the one-dimensional modeling of DEA to $\mathrm{CF}_{3} \mathrm{Cl}^{29,30,34}$ This multidimensional aspect of DEA will have to be included in future theoretical analyses of DEA to polyatomic molecules with the aim of obtaining a quantitative description of the DEA process.

Another possible reason for the substantial difference between $E_{B}$ and $E_{a}$ is the uncertainty in the definition of the reaction barrier. The anion curve presented in Fig. 1 is the diabatic curve corresponding to the energy of the lowest unoccupied orbital in the neutral molecule. This is not the eigenenergy of the fixed-nuclei electronic Hamiltonian for the anion. The latter corresponds to the adiabatic energy whose value is shifted relative to the diabatic value due to the interaction of the bound electronic state with the continuum. In the presence of a significant long-range electronmolecule interaction, the top of the adiabatic curve is significantly lower than the energy of the crossing point between the neutral and diabatic states. ${ }^{32}$ Although the adiabatic curve cannot be directly related to the DEA process, we should expect that the effective activation energy is lower than $E_{C}$ calculated from the diabatic curve.

To get further information on how the difference be-

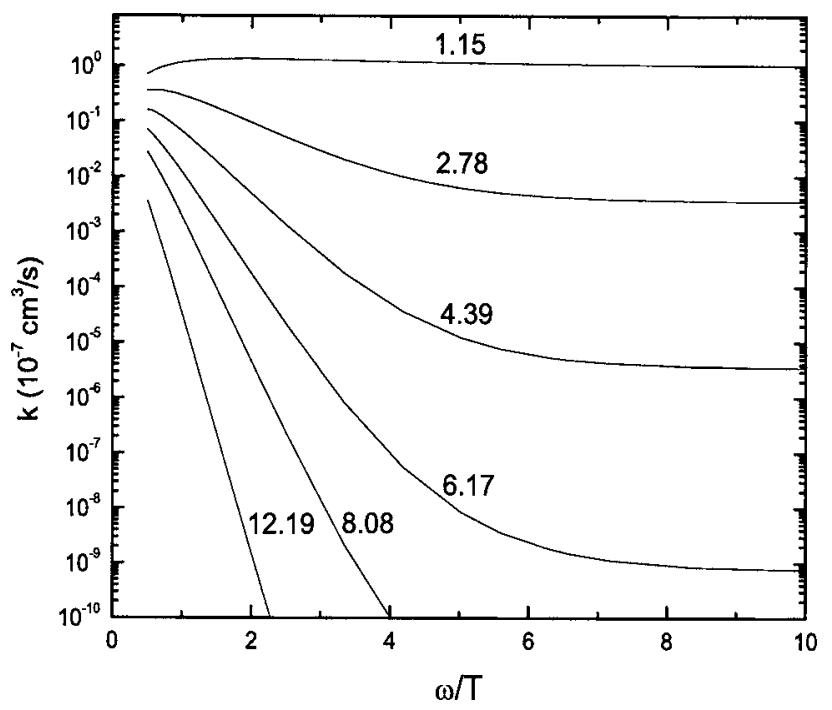

FIG. 7. DEA rate coefficients for the six exothermic cases listed in Table III. The vibrational frequency is fixed at $\omega=43.3 \mathrm{meV}$ and the barrier energy $E_{B}$ ranges from 50 to $528 \mathrm{meV}$. The corresponding ratios $E_{B} / \omega$ are listed in the figure next to the associated curve $k(\omega / T)$. tween $E_{B}$ and $E_{a}$ and the ratios $E_{a} / E_{B}$ are affected by the height of the barrier, we kept $\omega=43.3 \mathrm{meV}$ constant and varied $E_{C}$ and likewise $E_{B}$ by simply shifting the anion potential curve to large distances by amounts $\rho_{s}$ (see Table III), as illustrated in Fig. 1(b). In this way, the values for $E_{C}$ and $E_{B}$ as well as the vertical attachment energy (VAE) increase with rising $\rho$ while the exothermicity remains unchanged. Table III lists the values of VAE, $E_{B} / \omega$, and the activation energies $E_{a}$ deduced from the Arrhenius plots for the calculated rate coefficients $k(T)$ which are shown in Fig. 7. The functions $k(\omega / T)$ in Figs. 6 and 7 are observed to be quite similar for (nearly) the same parameters $E_{B} / \omega$. The values for $E_{a} / E_{B}$ are found to rise from 0.43 for $E_{B} / \omega=2.78$ to 0.79 for $E_{B} / \omega$ $=12.19$, the latter case being the "most classical."

\section{v. CONCLUSIONS}

Model calculations presented in this paper show that the Arrhenius equation for description of the DEA processes should be used with caution. For endothermic DEA processes and favorable curve crossing, the Arrhenius equation has a rather wide range of validity limited by high temperatures. The upper temperature limit depends on the threshold energy $E_{t}$ and becomes quite low for low $E_{t}$ and low vibrational frequency $\omega$. The activation energy in the Arrhenius equation is close to $E_{t}$.

In the case of exothermic DEA processes with an intermediate barrier, the range of validity of the Arrhenius equation is also limited toward low temperatures where the rate coefficient as a function of $T$ becomes flat. The activation energy $E_{a}$ is found to be lower than the reaction barrier $E_{B}$, and the relative difference $\left(E_{B}-E_{a}\right) / E_{B}$ decreases toward higher $E_{B}$. This behavior is partly due to the relatively slow growth of the partial rate coefficient $k\left(n, T_{e}\right)$ with rising $n$ for levels $n$ just below the barrier. The other reason for the difference between $E_{a}$ and $E_{B}$ is the uncertainty in the definition of the reaction barrier due to the long-range electronmolecule interaction the adiabatic anion curve peaks at lower energies than the diabatic curve. This effect is more significant for stronger long-range contributions to the electronmolecule interaction.

\section{ACKNOWLEDGMENTS}

The authors are grateful to G. A. Gallup, P. D. Burrow, and J. Troe for comments on the manuscript and M.-W. Ruf for providing the file of Fig. 2 and for helpful remarks. I.I.F. thanks the Fachbereich Physik for their hospitality during his recent visits to TU Kaiserslautern where most of this work was done. This work has been supported by the Deutsche Forschungsgemeinschaft (No. Ho 427/29) and by the U.S. National Science Foundation under Grant Nos. PHY0354688 and PHY-0652866.

${ }^{1}$ S. Arrhenius, Z. Phys. Chem. 4, 226 (1889), translated into English in Selected Readings in Chemical Kinetics, edited by M. H. Back and K. J. Laidler (Oxford, NY, (1967).

${ }^{2}$ Encyclopaedia Britannica (Encyclopedia Britannica, Inc., Chicago, IL, 2007) (http://www.britannica.com/eb/article-9009619).

${ }^{3}$ H. Hotop, M.-W. Ruf, M. Allan, and I. I. Fabrikant, Adv. At., Mol., Opt. Phys. 49, 85 (2003).

${ }^{4}$ W. E. Wentworth, R. George, and H. Keith, J. Chem. Phys. 51, 1791 
(1969).

${ }^{5}$ F. C. Fehsenfeld, J. Chem. Phys. 53, 2000 (1970).

${ }^{6}$ C. L. Chen and P. J. Chantry, J. Chem. Phys. 71, 3897 (1979).

${ }^{7}$ E. Alge, N. G. Adams, and D. Smith, J. Phys. B 17, 3827 (1984).

${ }^{8}$ S. M. Spyrou and L. G. Christophorou, J. Chem. Phys. 82, 2620 (1985).

${ }^{9}$ Z. Lj. Petrovic and R. W. Crompton, J. Phys. B 20, 5557 (1987).

${ }^{10}$ D. Smith and P. Spanel, Adv. At., Mol., Opt. Phys. 32, 307 (1994).

${ }^{11}$ R. G. Levy, S. J. Burns, and D. L. McFadden, Chem. Phys. Lett. 231, 132 (1994).

${ }^{12}$ T. M. Miller, A. E. Stevens, and J. F. Paulson, J. Chem. Phys. 100, 8841 (1994).

${ }^{13}$ P. Spanel, S. Matejcik, and D. Smith, J. Phys. B 28, 2941 (1995).

${ }^{14}$ S. Matejcik, G. Senn, P. Scheier, A. Kiendler, A. Stamatovic, and T. D. Maerk, J. Chem. Phys. 107, 8955 (1997).

${ }^{15}$ J.-L. Le Garrec, O. Sidko, J. L. Queffelec, S. Hamon, J. B. A. Mitchell, and B. R. Rowe, J. Chem. Phys. 107, 54 (1997).

${ }^{16}$ R. S. Wilde, G. A. Gallup, and I. I. Fabrikant, J. Phys. B 33, 5479 (2000).

${ }^{17}$ M. Braun, F. Gruber, M.-W. Ruf, S. V. K. Kumar, E. Illenberger, and H. Hotop, Chem. Phys. 329, 148 (2006).

${ }^{18}$ S. Marienfeld, T. Sunagawa, I. I. Fabrikant, M. Braun, M.-W. Ruf, and H. Hotop, J. Chem. Phys. 124, 154316 (2006).

${ }^{19}$ M. Braun, I. I. Fabrikant, M.-W. Ruf, and H. Hotop, J. Phys. B 40, 659 (2007).

${ }^{20}$ J. Troe, T. M. Miller, and A. A. Viggiano, J. Chem. Phys. 127, 244304
(2007).

${ }^{21}$ L. G. Christophorou and J. K. Olthoff, J. Phys. Chem. Ref. Data 29, 267 (2000).

${ }^{22}$ S. H. Alajajian and A. Chutjian, Phys. Rev. A 37, 3680 (1988).

${ }^{23}$ D. Klar, M.-W. Ruf, I. I. Fabrikant, and H. Hotop, J. Phys. B 34, 3855 (2001).

${ }^{24}$ K. Houfek, M. Čižek, and J. Horaček, Phys. Rev. A 66, 062702 (2002).

${ }^{25}$ A. Schramm, I. I. Fabrikant, J. M. Weber, E. Leber, M.-W. Ruf, and H. Hotop, J. Phys. B 32, 2153 (1999).

${ }^{26}$ L. G. Christophorou and J. K. Olthoff, J. Phys. Chem. Ref. Data 29, 553 (2000).

${ }^{27}$ S. Marienfeld, I. I. Fabrikant, M. Braun, M.-W. Ruf, and H. Hotop, J. Phys. B 39, 105 (2006).

${ }^{28}$ D. M. Pearl, P. D. Burrow, I. I. Fabrikant, and G. A. Gallup, J. Chem. Phys. 102, 2737 (1995).

${ }^{29}$ I. Hahndorf, E. Illenberger, L. Lehr, and J. Manz, Chem. Phys. Lett. 231, 460 (1994).

${ }^{30}$ R. S. Wilde, G. A. Gallup, and I. I. Fabrikant, J. Phys. B 32, 663 (1999).

${ }^{31}$ T. F. O’Malley, Phys. Rev. 155, 59 (1967).

${ }^{32}$ W. Domcke, Phys. Rep. 208, 97 (1991).

${ }^{33}$ I. I. Fabrikant, Phys. Rev. A 43, 3478 (1991).

${ }^{34}$ T. Beyer, B. M. Nestmann, and S. D. Peyerimhoff, J. Phys. B 34, 3703 (2001). 